\title{
Modulus of Rupture: Size EFfect due to Fracture INITIATION IN BOUNDARY LAYER
}

\author{
By Zdeněk P. Bazant,' Fellow, ASCE, and Zhengzhi Li
}

\begin{abstract}
Aвstract: The modulus of rupture of concrete, which characterizes the bending strength of unreinforced beams, is known to depend on the beam size. Because there is no large stable growth of a crack before the maximum load is reached, this size effect, unlike that in many other types of failure of concrete structures, cannot be explained by energy release due to fracture. Rather, this size effect must be explained by the fact that distributed microcracking and slips with strain softening take place in the boundary layer of the beam before the maximum load is reached. The beam is considered to fail before any macroscopic cracks are formed. A simple formula describing the size effect is derived. Asymptotic analysis of the strain softening in the boundary layer shows that the excess of the modulus of rupture over the direct tensile strength is inversely proportional to the beam depth and proportional to the thickness of the boundary layer, which itself is approximately proportional to the maximum aggregate size. The proposed formula agrees with the existing experimental data quite well. The formula is further generalized to describe the effect of the gradient of normal strains near the concrete surface. Finally, it is shown that approximate analysis of the size effect by linear elastic fracture mechanics yields similar formulas. Those formulas, however, have some questionable features; for example, they indicate the size effect magnitude depends on the span-to-depth ratio of the beam, which has not been observed in experiments.
\end{abstract}

The modulus of rupture, which characterizes the apparent tensile strength of concrete beams, has for a long time been known to depend on the size of the beam. Initially, it was thought that the source of this dependence is statistical, caused by randomness of the intrinsic material strength. However, this explanation ignores the stress redistributions caused by cracking prior to the maximum load. This is a deterministic effect, which must be taken into account before statistical analysis. Furthermore, it is questionable (and not indicated by the available test data) that the statistical theory predicts the size effect to be much weaker in three-point-bend beams than in four-point-bend beams (because the zone of maximum stress is much shorter in the former). It is of course likely that there is at least some statistical size effect, but this size effect is probably small. Anyway, it would have to be included in the analysis only after the deterministic size effect is taken into account. However, as we will see, the deterministic size effect alone can explain the existing test data adequately.

The basic explanation of the size effect on the modulus of rupture is to be found in the theory of quasi-brittle fracture, describing materials of heterogeneous microstructure in which the formation of distinct fractures is preceded by distributed cracking. The failure of a beam begins by distributed cracking that develops in a boundary layer. The thickness of this layer for different beam sizes is about the same, provided the same concrete is considered. Hillerborg et al. (1976) showed by numerical calculations that the stress distribution at the peak load has a maximum that lies at a certain distance from the tensile face. This distance is determined by the softening stress-displacement relation of the cohesive (fictitious) crack model. Hillerborg et al. also demonstrated that numerical calculations based on the cohesive crack model can match the published test results on the influence of beam size. This is of course logical to expect, since the predictions of fracture mechanics generally exhibit a size effect.

The calculations of Hillerborg relied on a relatively sophisticated model-the fictitious crack model. The solution had to be obtained numerically, by finite elements. The purpose of the present paper is to describe the effect of beam size (or, more generally, of the strain gradient) on the modulus of rupture by a short formula, the use of which would be simpler than a finiteelement solution.

\footnotetext{
'Walter P. Murphy Prof. of Civ. Engrg., Northwestern Univ., Evanston. IL 6()208

'Grad. Res. Asst., Dept. of Civ. Engrg., Northwestern Univ., Evanston. IL.

Note. Associate Editor: Steven L. McCabe. Discussion open until September 1, 1995. To extend the closing date one month, a written request must be filed with the ASCE Manager of Journals. The manuscript for this paper was submitted for review and possible publication on June 1, 1993. This paper is part of the Journal of Structural Engineering, Vol. 121, No. 4, April, 1995. CASCE, ISSN 0733-9445/95/00(04-0739-0746/\$2.010 + \$.25 per page. Paper No. 6287.
} 


\section{DERIVATION OF FORMULA BASED ON DISTRIBUTED MICROCRACKING}

The size effect caused by formation of the fracture process zone in the boundary layer is the principal reason for the difference between the direct tensile strength $f_{t}^{\prime}$ and the so-called modulus of rupture

$$
f_{r}=6 M_{1} / b h^{2}
$$

$f$. represents the apparent maximum stress in the cross section calculated from the bending theory; $M_{u}=$ ultimate bending moment, obtained on a simply supported beam of a constant rectangular cross section; and $h=$ depth and $b=$ width of the cross section [Fig. 1(a)]. Eq. (1) presumes a linear stress distribution throughout the cross section [Fig. 1(d)]. In reality, near the tensile face, there must be a layer of some thickness $l_{f}$ [Fig. 1(c)] in which the stress is reduced [segment $\overline{13}$ in Fig. 1(e)] due to cracking. To describe the stress reduction, there are now two possible hypotheses.

One possible hypothesis is that, up to the peak load, the cracking remains distributed, being stabilized against localization by the restraint provided by uncracked concrete [concrete above line $\overline{71}$ in Fig. 1(e)]. In that case, the reduction of stress due to cracking [segment $\overline{23}$ in Fig. $1(\mathrm{e})$ ] is properly described according to continuum damage mechanics. This means that, for uniaxial stress, the effect of cracking on stress can be approximately described by a stress-strain diagram with postpeak strain softening. For the sake of simplicity, we may consider this diagram to be approximately triangular, characterized by linear softening of slope $E_{t}(<0)$, direct tensile strength $f_{t}^{\prime}$, and Young's elastic modulus $E$ of concrete, as shown in Fig. 1(b). The consequence of the postpeak softening is that the stress distribution in Fig. 1(d) is changed to that in Fig. 1(e). Near the tensile face there is a layer of reduced stress (segment 13) up to a certain unknown depth $l_{f}$, with stress reduction given by $k l_{\text {f }}$ at the tensile face. From the fracture mechanics viewpoint, the thickness $l_{f}$ of the boundary layer represents the effective depth of the fracture process zone at maximum load.

Another possible hypothesis is that there is no distributed cracking but a vertical crack growing from the tensile face. As is known from fracture mechanics of concrete [see e.g. ACI Committee 446 (1992)], a crack in concrete must be considered as a cohesive crack transmitting crackbridging (cohesive) stresses, as described by the fictitious crack model of Hillerborg et al. (1976). The bridging stress $\sigma$ is a function of the crack opening displacement $\omega$, which may be approximately considered as linear, characterized by stress $\sigma=f_{t}^{\prime}$ at $z=0$ and stress $\sigma=0$ at a certain displacement $r_{f}$ signifying complete separation. The length of the crack is determined from the condition that the combined stress intensity factor due to both the applied load and the crack-bridging stress is zero.

In the present study, we adopt the former hypothesis, assuming that, up to the peak load, the cracking in the layer of thickness $l_{f}[\mathrm{Fig} .1(\mathrm{c})]$ is forced to remain distributed because of the restraint provided by the uncracked concrete [concrete above the line $\overline{71}$ in Fig. 1(e)], which stabilizes the cracking against localization (Bažant and Cedolin 1991). After the peak load, the cracking of course localizes into one major crack, but we do not need to analyze the postpeak behavior. The first hypothesis is also simpler. It does not require the use of fracture mechanics and thus it blends better with the philosophy of American Concrete Institute (ACI) code. Both hypotheses, however, may be expected to yield approximately equivalent results for the threepoint-bend beam specimens. The crack opening $z$ in the cohesive (fictitious) crack model is approximately equal to the average cracking strain $\bar{\varepsilon}_{c r}$ times the effective width $w_{c}$. [Fig. 1(a)] of the cracking zone, which means that $\bar{\varepsilon}_{c r} w_{c}={ }^{\prime}$, where $\bar{\varepsilon}_{c r} \cong\left(f_{t}^{\prime}-\sigma\right)\left(E^{-1}-E_{\prime}^{-1}\right)$. Profile $\because$ is considered as linear, which is often an acceptable approximation.

From the condition of plane cross sections, we obtain, for $l_{f}<<h / 2$, the approximation

$$
k=\frac{2 f_{i}^{\prime}}{h}\left(1-\frac{E_{t}}{E}\right)
$$
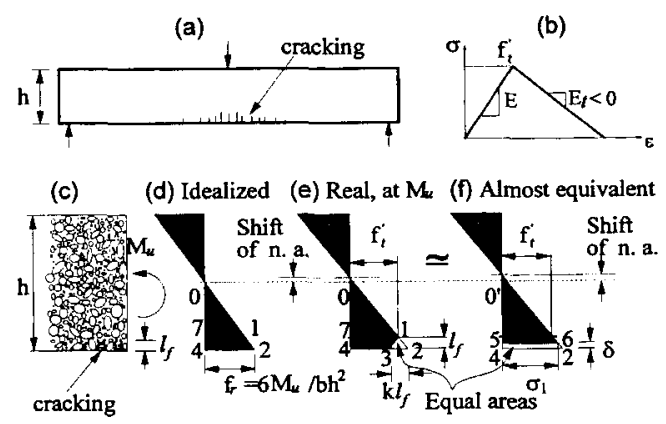

FIG. 1. Stress and Strain Distributions in Critical Cross Section as Influenced by Boundary Layer and Deduced from Strain-Softening Analysis 
where $E_{t}<0$. To solve the problem according to the bending theory, it is now convenient to replace the triangular area 1231 in Fig. 1(e) by an equal trapezoidal area 45624 of thickness $\delta$, shown in Fig. 1(f). If these two areas are equal and if $l_{f} \ll h$, the bending moment remains approximately the same (except for a higher order error). The replacement of the areas yields a linear stress-strain diagram with a shifted neutral axis and maximum tensile stress $\sigma_{1}=6 M_{u} l$ $b(h-\delta)^{2}$. From the condition of equal areas, $k l_{f}^{2} / 2=\sigma_{1} \delta$, we have

$$
\delta=\frac{k l_{f}^{2}}{2 \sigma_{1}} \approx \frac{k l_{f}^{2}}{2 f_{i}^{\prime}}
$$

According to the definition of the modulus of rupture $f_{r}, M_{u}=f_{r} b h^{2} / 6$, which yields

$$
\sigma_{1}=\frac{f_{r} h^{2}}{(h-\delta)^{2}}
$$

At the same time, because of the approximate similarity of triangles 0170,0240 , and 0$)^{\prime} 560^{\prime}$ in Fig. $1(\mathrm{~d}-\mathrm{f})$, we have $\sigma_{1} /[(h / 2)-\delta]=\overline{56} / \overline{0^{\prime} 5} \approx \overline{71} / \overline{07}=f_{l}^{\prime} /\left[(h / 2)-l_{f}\right]$ or $\sigma_{1}=f_{t}^{\prime}(1-2 \delta / h) /$ $\left(1-2 l_{f} / h\right)$. Setting this equal to $(4)$, we obtain

$$
\frac{f_{r}}{f_{i}^{\prime}}=\frac{1-(2 \delta / h)}{1-\left(2 l_{f} / h\right)} \frac{(h-\delta)^{2}}{h^{2}}
$$

This expression can be simplified for $\delta<<h$ and $l_{f}<h / 2$ by noting the Taylor series expansion $\left(1-2 l_{f} / h\right)^{-1}=1+2 l_{f} / h+4 l_{f}^{2} / h^{2}+\cdots$. Introducing also the expressions (3) and (2), we get

$$
\frac{f_{r}}{f_{i}^{\prime}}=1+2\left(\frac{l_{f}}{h}\right)+\frac{4 E_{t}}{E}\left(\frac{l_{l}}{h}\right)^{2}+\cdots
$$

Assuming that the fracture process zone is short compared to the half-depth of the beam, i.e., $l_{f} \ll h / 2$, we may drop the terms higher than linear. Thus we obtain the following simple result:

$$
\frac{f_{r}}{f_{t}^{\prime}}=1+\frac{2}{h} l_{f}
$$

which gives the first approximation of the size effect on the ratio of the modulus of rupture to the direct tensile strength.

\section{ANALYSIS AND VERIFICATION OF PROPOSED FORMULA}

According to the ACI Standard [ACI Committee $318(1992)], f_{t}^{\prime}=6 \sqrt{f_{c}^{\prime}}$ and $f_{r}=7.5 \sqrt{f_{c}^{\prime}}$, where $f_{c}^{\prime}=$ standard cylindrical compression strength. This means that ACI assumes $f_{r} / f_{i}^{\prime}=$ 1.25 , which implies that $l_{f}=h / 8$ in (7). This value of $l_{f}$ is of the same order of magnitude as the maximum aggregate size $d_{a}$ in typical test beams, which does not seem unreasonable.

Although $f_{r}=1.25 f_{t}^{\prime}$ is not a bad estimate of the experimental values for normal beam sizes and normal aggregates, very different ratios are observed for unusual beam depths $h$ and unusual aggregate sizes $d_{a}$. Formula (7) provides a better estimate, provided that, of course, the depth $l_{f}$ of the boundary layer of cracking at the maximum load is known. An important property is that this formula gives the ratio of these two tensile strength measures as a function of $h$, and thus also of $d_{a}$ if $l_{f}$ is assumed roughly proportional to $d_{a}$. This property is born out by the test data existing in the literature, including Reagel and Willis (1931), Kellerman (1932), Wright and Garwood (1952), Nielsen (1954), Lindner and Sprague (1953), Walker and Bloem (1957), Mal'cov and Karavaev (1968), and Avram (1981). Comparisons of (7) with these eight data sets and their regression lines are shown in Figs. 2 and 3. Because most authors reported only $f$, but not $f_{i}^{\prime}$, the $f_{i}^{\prime}$ values have been obtained from the regressions. For the data of Mal'cov, only the ratios $f_{r} / f_{i}^{\prime}$, were reported, and the typical concrete tensile strength $f_{t}^{\prime}=500$ psi was assumed for these data. Further extensive data on $f_{r}$ were presented by Mayer (1967), but they have an insufficient range of specimen sizes.

The value of $l_{f}$ cannot be expected to be the same as the effective length $c_{f}$ of the fracture process zone in front of a very large crack. Both, however, may be expected to be roughly proportional to the maximum aggregate size $d_{a}$ and represent a material property. The probable approximate proportionality of $l_{f}$ to $d_{c}$ cannot be verified from the existing data, because most experimenters have not reported the value of $d_{a}$. It might be possible to determine $l_{\text {f }}$ theoretically, by means of an energy argument of the type explained in Sec. 12.6 of Bažant and Cedolin (1991) [or a refined argument of this type made to determine the maximum depth of the distributed cracking zone in sea ice; see Bažant and Li (1993)]. Such an investigation, however, is beyond the scope of this paper.

Because the data were obtained on very different concretes, they are first fitted by (7) individually. This is easily accomplished by linear regression; see Fig. 2. Subsequently, in order to show how well the formula fits the data overall, all the plots are combined in Fig. 3 , in which 

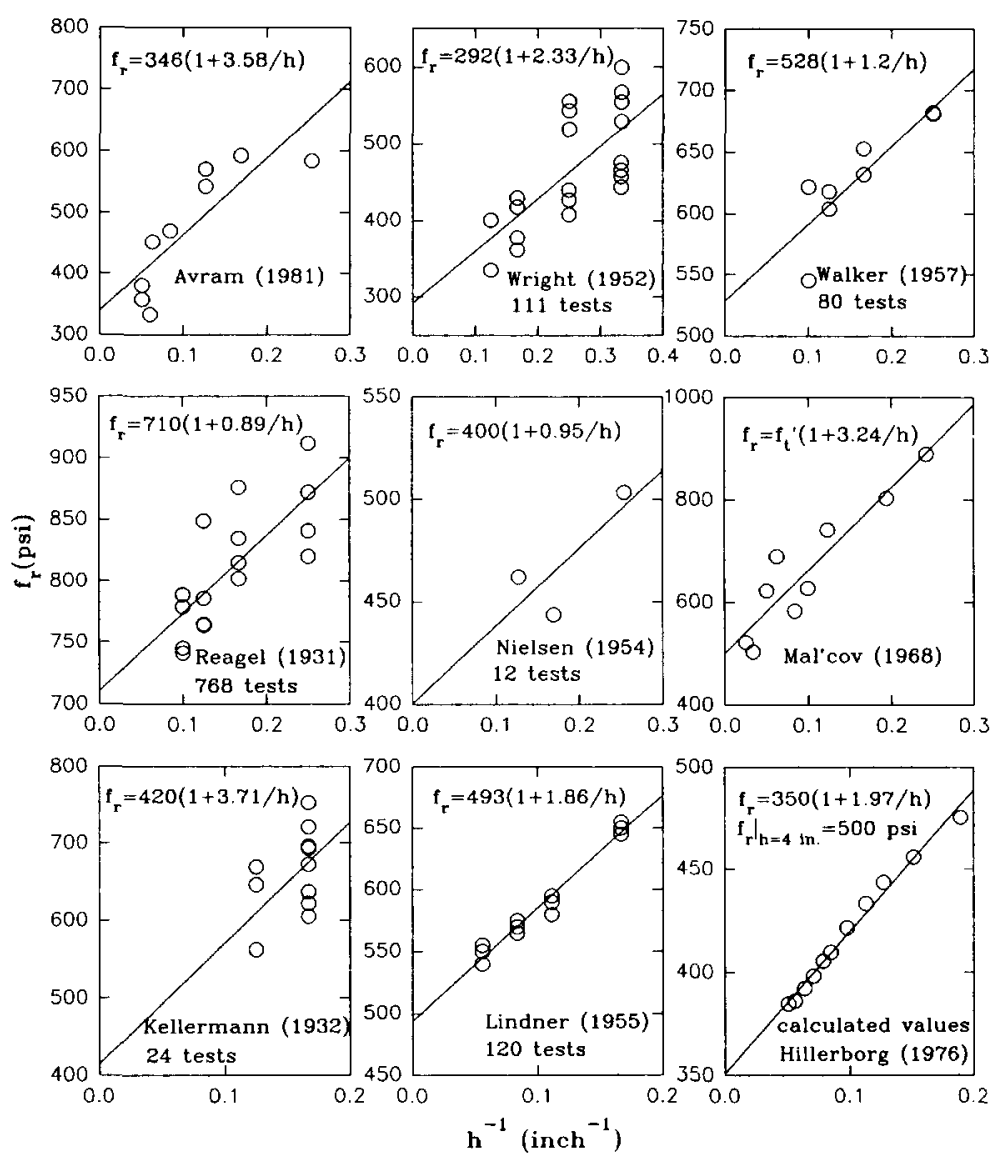

FIG. 2. Optimum Fits of Proposed Formula to Test Results from Literature, Obtained by Linear Regression

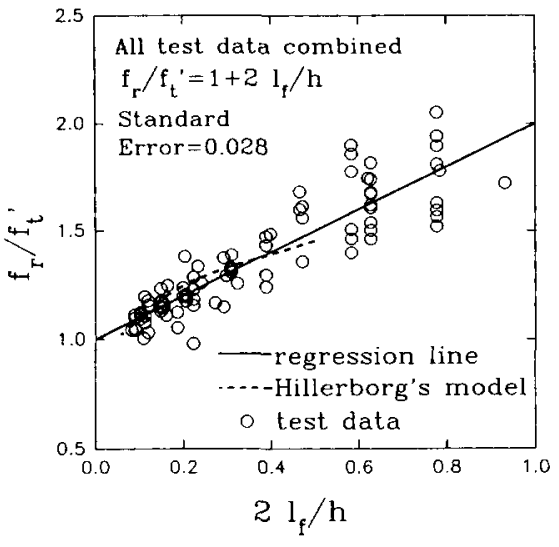

FIG. 3. Comparison of Proposed Formula (Straight Solid Line) with Test Results from Literature, and with Prediction of Cohesive Crack Model by Hillerborg et al. (1976)
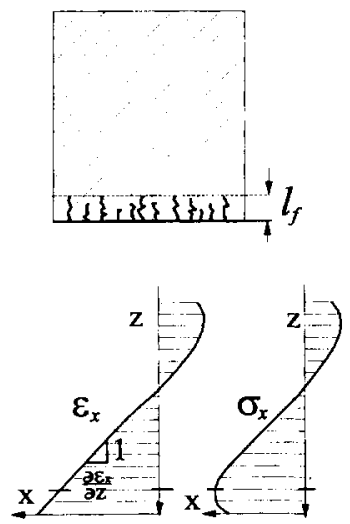

FIG. 4. Depth of Cracking at Maximum Load and Stress Distribution in Presence of Strain Gradient

the coordinate is $X=2 l_{f} / h$, where all the $l_{f}$ values are taken from the individual optimum fits in Fig. 2.

The same data were used in a pioneering study by Hillerborg et al. (1976). They showed that the difference between the modulus of rupture and the direct tensile strength can be explained by nonlinear fracture mechanics, particularly the cohesive (or fictitious) crack model. Their study, however, was strictly numerical; they did not attempt to derive any formula for $f_{\prime}^{\prime} / f_{\prime}^{\prime}$. Hillerborg et al.'s calculations are shown in Fig. 3 by the dashed curve. They are seen to be also in good agreement with the data, even though the model, consisting of a single cohesive (fictitious) crack at midspan, was different. This is not surprising, since, as already commented, the stress distribution at the cohesive crack is similar to that in Fig. 1(e).

However, there are differences from Hillerborg's model. The three-point loading used causes 
the bending moment to decay rapidly from midspan. This limits the length of the cracking zone, causing it to be well represented by a single cohesive crack. If four-point loaded beams were used, or if the length-to-depth ratio were unusually large, fitting of the results with the cohesive crack model might require considering several cohesive cracks. The spacing of these cracks cannot be determined from the cohesive crack model as defined by Hillerborg, and would have to be given. Especially, it would be necessary to ensure that the spacing cannot be less than a certain minimum [see ACI Committee 446 (1992)]. The idea that a certain minimum crack spacing ought to be introduced as an additional characteristic of the cohesive (fictitious) crack model has been proposed in Bažant (1986) and has been theoretically supported in more detail by Planas and Elices (1992). To decide these questions experimentally, it would be necessary to compare the values of the modulus of rupture not only for different beam sizes, but also for other span-to-depth ratios and for four-point bending, and compare the deflections as well.

Note that formula (7) does not involve the strain-softening modulus $E_{t}$ [this modulus affects only the higher-order small terms in (6)]. This provides a useful simplification, since the value of $E_{i}$ is quite uncertain. The only case in which the value of $E_{z}$ would affect (and also invalidate) this formula is the case of a sudden stress drop, $\left|E_{t}\right| \rightarrow \infty$. But that is not the real behavior of concrete; it is only an approximation for the combined behavior of the fracture process zone and the structural element (or finite element), in which the sudden stress drop is only an apparent characteristic representing an instability caused by energy release [chapter 13 in Bažant and Cedolin (1991)].

The simplicity of formula (7) makes it possible to determine the direct tensile strength $f_{i}^{\prime}$ and the boundary layer thickness $l_{f}$ by the linear regression plots shown in Fig. 2 . It is simply necessary to test similar beams of a sufficiently large range of depths $h$, made of the same concrete. Fig. 2 also shows the data points from the aforementioned published studies. Fig. 3 shows the regression of the eight groups of test data normalized by their $l_{f}$ and $f_{t}^{\prime}$ values. It is seen that the deviations from the regression line are quite acceptable.

It may be noted that a formula similar to (7) has been proposed on the basis of different arguments by Zhu (1990). His formula reads $f_{r} / f_{i}^{\prime}=1+2\left(s_{1} / f_{t}^{\prime}\right) l_{1} / h$, where $s_{1}$ is the normal stress at the tensile face. For the special case $s_{1}=f_{r}^{\prime}$, this formula coincides with (7). The $f$. value, according to $Z$ hu, can be obtained for a certain critical value of $l_{1}$, which is similar to $l_{f}$ but is not considered by $\mathrm{Zhu}$ as a material property. A formula similar to (7) was also proposed on a purely empirical basis by Mal'cov and Karavaev (1968). Finally, it may be remarked that (7) coincides with the first two terms of a general asymptotic expansion of the size effect on nominal strength for failures at crack initiation, which was derived by Bažant (1994) by dimensional analysis.

\section{GENERALIZATION: STRAIN GRADIENT EFFECT}

From the foregoing analysis it is clear that the size effect on the modulus of rupture is caused by the strain gradient $\partial \varepsilon_{x} / \partial z$, where $z$ is the coordinate normal to the face of beam and $\varepsilon_{x}$ is the normal strain in direction $x$ parallel to the face (Fig. 4). At maximum load, the strain gradient in the beam (unreinforced) is approximately $\partial \varepsilon_{x} / \partial z=2 f_{z}^{\prime} / h E$, from which $h=2 f_{i}^{\prime} /\left(E \partial \varepsilon_{x} / \partial z\right)$. Substituting this into (7), we obtain the general formula

$$
\frac{f_{r}}{f_{i}^{\prime}}=1+\frac{l_{f} E}{f_{i}^{\prime}} \frac{\partial \varepsilon_{x}}{\partial z} \text { if }>1, \text { else } 1
$$

where $f_{r}$ is now more generally interpreted as the strength limit, at the surface for normal stress $\sigma_{x}$ parallel to the surface, at any strain distribution in the body, linear or curved, caused by a combination of moment and normal force or otherwise; $\partial \varepsilon_{x} / \partial z$ is taken positive when $\varepsilon_{x}$ increases toward the surface. The limitation that $f_{r} / f_{t}^{\prime}>1$ in (8) is due to the fact that for the case $\partial \varepsilon_{x} /$ $\partial z>0$ (strain increasing away from the surface) it would be unreasonable to expect a strength increase because, if a crack forms, it must in that case quickly propagate deep into the body.

Note, however, that if the maximum load is reached only after the crack emanating from an unnotched surface becomes much longer than $l_{f}$, then (8) and (7) are inapplicable and the size effect due to energy release operates.

\section{CONCLUSIONS}

1. The hypothesis that cracking remains distributed up to the maximum load and is thus characterized by a strain-softening stress-strain diagram leads to the conclusion that the maximum of the stress distribution in the cross section at maximum load occurs at a certain finite distance from the tensile face, which is approximately a material property related to the maximum inhomogeneity size (maximum aggregate size).

2. The formula resulting from this hypothesis predicts that the modulus of rupture depends on the beam size or on the magnitude of the strain gradient at the surface (as well as other factors). The first-order approximation of the size effect is independent of the 


\section{ACKNOWLEDGMENT}

strain-softening slope of the stress-strain diagram; it depends only on the ratio of the boundary layer thickness to the beam depth.

3. The formulation also implies the hypothesis that localized fractures appear only after the maximum load is reached. Consequently, analysis according to linear elastic fracture mechanics cannot give realistic results. This analysis, too, predicts the ratio of the modulus of rupture to the direct tensile strength to depend on the beam size. However, it predicts this ratio to depend also on the ratio of the beam span to the depth, which is not substantiated by the existing test results. Further it predicts a similar size effect to occur in direct tension tests, which is likewise not corroborated by the existing test results.

4. Predictions of the proposed simple formula agree well with the existing test results, as far as the random scatter permits it to say. The agreement with the test data is as good as that of the numerical calculations of Hillerborg et al. using the cohesive (fictitious) crack model.

5. Due to the linear form of the proposed formula, the thickness of the boundary layer can be determined by linear regression of the test results on the size dependence of the modulus of rupture. The proposed formula can also be used as an indirect method to predict tensile strength from bending tests.

Partial financial support under AFOSR91-(0140 grant to Northwestern University, monitored by Dr. Jim Chang. is gratefully acknowledged. The collection and analysis of experimental results was partially supported by the Center for Advanced Cement-Based Materials at Northwestern University.

\section{APPENDIX I. COMMENTS ON ALTERNATIVE LEFM APPROACH}

According to linear elastic fracture mechanics (LEFM), the initiation of crack growth from a smooth surface would require an infinite load because for a finite load the stress intensity factor is zero as the crack length tends to zero. Obviously, for LEFM to be meaningful at all, it is necessary to assume that the fracture growth starts from a crack (or initial flaw) of a certain finite initial length, $a=a_{0}$. A crack of this length (a macrocrack, rather than microscopic flaws) forms by localization of distributed cracking. This cannot happen before the maximum load (Fig. 5), because LEFM predicts the load on beams to decrease with increasing $a$. Thus the initial crack (a macrocrack) can form either right after the maximum load or later. In the former case, the maximum load can be calculated from the condition of propagation of the initial macroscopic crack. The basic assumption is that the length $a_{0}$ of this crack is, at least approximately, a material property. In other works, $a_{0}$ is the same for specimens of various sizes, and also for bending specimens [Fig. 6(a)] and direct tension specimens [Fig. 6(b)].

According to LEFM, the stress intensity factor of the crack in a three-point-bend [Fig. 6(a)] specimen of $\operatorname{span} L=4 h$ is $K_{1}=\sigma \sqrt{\pi a_{0}} F_{b}(\alpha)$ where $\alpha=a_{0} / h ; \sigma=6 M / b h^{2} ;$ and $F_{l}(\alpha)=$ $\pi^{-1 / 2}\left[1.99-\alpha(1-\alpha)\left(2.15-3.93 \alpha+2.72 \alpha^{2}\right)\right](1+2 \alpha)^{-1}(1-\alpha)^{-3 / 2}$ (Tada et al. 1985). In a direct tension specimen, fracture initiates by a one-sided crack [Fig. 6(b)], for which $K,=$ $\sigma \sqrt{\pi a_{0}} F_{t}(\alpha)$ with $F_{t}(\alpha)=1.12-0.23 \alpha+10.6 \alpha^{2}+\cdots$ (Tada et al. 1985). Considering that the direct tensile strength $f_{t}^{\prime}$ corresponds to $h \rightarrow \infty$, i.e. $f_{t}^{\prime}=f_{t \%}^{\prime}$, we have $F_{t}(\alpha)=F_{t}(0)=1.12$, and setting $K_{l}$ for the beam of size $h$ equal to $K_{I c}$, we have $K_{t c}=f_{r} \sqrt{\pi a_{0}} F_{b}(\alpha)=f_{l}^{\prime} \sqrt{\pi a_{0}} F_{r}(0)$. Therefore, noting the binominal series expansion $(1-\alpha)^{3 / 2}=1-1.5 \alpha-2.625 \alpha^{2}-\cdots$, $f_{,} / f_{t}^{\prime}=1.12 / F_{b}(\alpha)=1.12 \sqrt{\pi}(1+2 \alpha)(1-\alpha)^{3 / 2} / Y=1.12 \sqrt{\pi}\left(1+0.5 \alpha+0.375 \alpha^{2}+\cdots\right) / Y$, where $Y=1.99-2.15 \alpha+6.08 \alpha^{2}+\cdots$. By polynomial division, we then get for $L / h=4$ the result

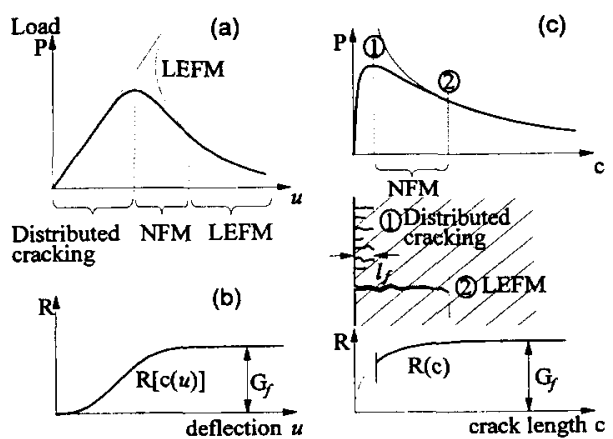

FIG. 5. (a) Load-Deflection Diagrams (b) Corresponding Variation of Energy Required for Crack Growth; (c) Variation of Load with Depth of Cracking or Length of Fracture
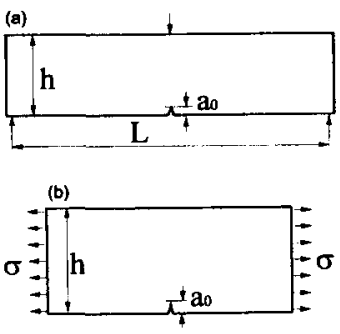

FIG. 6. Bending and Direct Tension Specimens Considered in LEFM Analysis 


$$
\frac{f_{r}}{f_{1 x}^{\prime}}=1+1.58 \frac{a_{0}}{h}-3.97\left(\frac{a_{0}}{h}\right)^{2}+\cdots \quad(\text { for } L / h=4)
$$

For a beam with $L / h=8$, we have $F_{b}(\alpha)=1.11-2.12 \alpha+7.71 \alpha^{2}-13.55 \alpha^{3}+14.23 \alpha^{4}$, and from $f_{r} / f_{t x}^{\prime}=1.12 / F_{b}(\alpha)$ we get by polynomial division

$$
\frac{f_{r}}{f_{i x}^{\prime}}=1+1.94 \frac{a_{0}}{h}-3.34\left(\frac{a_{0}}{h}\right)^{2}+\cdots \quad(\text { for } L / h=8)
$$

For the direct tension test, setting $K_{l}$ for size $h$ equal to $K_{l \cdot}=f_{t}^{\prime} \sqrt{\left.\pi a_{0}\right)} F_{l}(0)$, one gets

$$
\frac{f_{t}^{\prime}}{f_{i x}^{\prime}}=1+0.21 \frac{a_{0}}{h}-9.42\left(\frac{a_{0}}{h}\right)^{2}+\cdots
$$

However, the foregoing LEFM solutions invite several questions the answers to which are not clear at present:

1. First of all, it is unclear whether a sharp crack forms right after the maximum load or later. Most likely it depends on the size; for very large sizes the localization happens right at the peak load, and for very small sizes much later (this would also mean that $a_{\mathrm{t}}$, unlike $l_{\mathrm{f}}$, is not a constant).

2. The size dependence is predicted to be different for different $L / h$, while according to (7) it is the same. But no clear effect of $L / h$ has been observed in experiments. It might be that a possible effect of $L / h$ has been obscured by inevitable statistical scatter. To clarify this point, it would be necessary to conduct a series of tests with both a large size range and a large range of $L / h$, using the same concrete.

3. The direct tensile strength is predicted, by the LEFM analysis, to be also size dependent . However, no clear size effect trend, such is that in Figs. 2 and 3, is known for $f_{r}^{\prime}$ (except perhaps for a statistical size effect). On the other hand, the type of argument that led to (7) predicts no size effect for $f_{\text {, }}^{\prime}$.

4. It does not seem quite realistic to assume that $K$, and $a_{0}$ are the same for different sizes. One must expect the effective $K_{I}$ to vary according to an $R$-curve; see [(Fig. 5(b)]. However, there are two difficulties with introducing the $R$-curve into the foregoing analysis: (1) The $R$-curves depend on the specimen shape; and (2) no effective $R$-curve for the start of a crack from a smooth surface is known.

For these reasons, the foregoing LEFM-based equations do not seem very realistic. The actual behavior is probably that sketched in Fig. $5(\mathrm{a}$ and c) showing that LEFM is approached only for larger deflections corresponding to a large crack [Fig. 5(c)], provided that the ligament is long enough. Right after the peak one would need to use nonlinear fracture mechanics on which we already commented. Nevertheless, it is interesting to know what LEFM would predict.

\section{APPENDIX II. REFERENCES}

ACI Committee 318. (1992). Building code requirements for reinforced concrete (ACI 318-89) (revised 1992) and Commentary-ACI 318R-89 (revised 1992). Am. Concrete Inst. (ACI), Detroit, Mich.

ACI Committee 446. (1992). "State-of-art-report on fracture mechanics of concrete: concepts, model and determination of material properties." Fracture mechanics of concrete structure, Z. P. Bažant, ed., Elsevier Appl. Sci., New York. N.Y., 4-144.

Avram, C. et al. (1981). Concrete strength and strains. Elsevier, New York, N. Y., 255-268.

Bažant, Z. P. (1986). "Mechanics of distributed cracking." Appl. Mech. Reviews ASME, 39(5), 675-705.

Bažant, Z. P. (1994). "Is size effect caused by fractal nature of crack surfaces'?" Rep. 94-10/C402i. Dept. of Civ.

Engrg., Northwestern Univ., Evanston, Ill.

Bažant, Z. P., and Cedolin. L. (1991). Stability of structures: elastic, inelastic, fracture and damage theories. Oxford University Press, New York, N.Y.

Bažant, Z. P., and Li, Y.-N. (1993). "Penetration through floating sea ice plate and size effect: simplified fracture analysis." J. Engrg. Mech. ASCE, 120(6), 1304-1321.

Fracture mechanics of concrete structure; Proc., 1st Int. Conf. (1992). Z. P. Bažant, ed., Elsevier, London. England.

Hillerborg, A., Modéer, M., and Petersson, P.-E. (1976). "Analysis of crack formation and crack growth in concrete by means of fracture mechanics and finite elements." Cement and Concrete Res., 6(6), $773-782$.

Kellerman, W. F. (1932). "Effect of size of specimen, size of aggregate and method of loading upon the uniformity of flexural strength tests." Public Roads, 13(11), 177-184.

Lindner, C. P., and Sprague, I. C. (1955). "Effect of depth of beams upon the modulus of rupture of plain concrete." ASTM Proc., Vol. 55, $1062-1083$.

Mal'cov, W. K., and Karavaev, A. (1968). "Abhängigkeit der Festigkeit des Betons auf Zug bei Biegung und ausmittiger Belastung von den Querschnittsabmessungen." Wissenschaftlihe Zeitschrift der Technischen Universität, Dresden, Germany, Vol. 6, 1545-1547 (in German).

Mayer, H. (1967). "Die Berechnung der Durchbiegung von Stahlbetonbauteilen." Deutscher Ausschuss für Siahlbeton, Vol. 194, Wilhelm Ernst \& Sohn, Berlin, Germany (in German).

Nielsen, K. E. C. (1954). "Effect of various factors on the flexural strength of concrete test beams." Mag. of Concrete Res., 15(Mar.). 105-114. 
Planas, J., Elices. M., and Guinea, G. V. (1993). "Cohesive cracks vs. nonlocal models: closing the gap." Int. J. Fracture. 63(2), 173-188.

Reagel, F. V., and Willis, T. F. (1931). "The effect of dimensions of test specim.'ns on the flexural strength of concrete." Public Roads, 12(2), 37-46.

Tada, H., Paris, P., and Irwin, G. (1985). The stress analysis of cracks handbook, 2nd Ed., Paris Productions Inc., Missouri.

Walker. S. and Bloem, D. L. (1957). "Studies of flexural strength of concrete-part 3: effects of variations in testing procedures." ASTM Proc., Vol. 57, 1122-1139.

Wright, P. J. F., and Garwood, F. (1952). "The effect of the method of test on the flexural strength of concrete." Mag. of Concrete Res., 11(Oct.), 67-76.

Zhu, Y. (1990). "The flexural strength for concrete beams without initial cracks." Proc., 8th European Congress of Fracture: Fracture Behavior and Design of Mat. and Struct., Vol. 2. D. Firrao, ed., Chameleon Press, Ltd., London, England, 599-60)4. 